\title{
Islam und Sozialkapital \\ Beispiele muslimischer Gruppierungen in Deutschland
}

Yasemin El-Menouar

\section{Einleitung}

Das Interesse der Öffentlichkeit am Islam besteht seit Jahren unverändert fort und wird durch die mediale Aufmerksamkeit hoch gehalten. Die Diskussionen in Deutschland kreisen meist um Themen, die sich mit der Religiosität von Muslimen und die dahinter vermutete Desintegration befassen. In kurzer Zeit sind zahlreiche Studien zu diesen Themenkomplexen realisiert worden, die zum Teil $\mathrm{zu}$ unterschiedlichen Ergebnissen kommen. ${ }^{1}$ Dies ist unter anderem auf die uneinheitliche Verwendung der Begriffe zurückzuführen. Was wird genau unter Integration verstanden?

Die neueren theoretischen Konzepte sind sich zumindest einig über das Ziel der Integration - oder neutraler ausgedrückt - über das Ziel einer erfolgreichen Eingliederung. Personen mit Migrationshintergrund sollen nach einer vollzogenen Eingliederung genauso stark bzw. genauso wenig von sozialer Ungleichgeit betroffen sein wie Einheimische, d.h., der Anteil von Arbeitslosen bspw. sollte sich zwischen Einheimischen und Personen mit Migrationshintergrund nicht unterscheiden. Die anteilsmäßige Verteilung der verschiedenen Bevölkerungsgruppen auf dem gesellschaftlichen Positionssystem sollte sich angleichen. ${ }^{2}$ Damit das erreicht werden kann, müssen Personen mit nichtdeutschen Wurzeln in angesehenen gesellschaftlichen Positionen vertreten sein.

Über die Wege, die zur erfolgreichen Integration führen, besteht nach wie vor Uneinigkeit zwischen den Autoren. Während nach Hartmut Esser - sein

\footnotetext{
${ }^{1}$ Vgl. exemplarisch Sonja Haug, Stefanie Müssig und Anja Stichs 2009; Karin Brettfeld und Peter Wetzels 2007; Wolfgang Frindte et al. 2011; Martina Sauer und Dirk Halm 2009.

${ }^{2}$ Vgl. Jutta Aumüller 2009; Silke Hans 2010.
} 
Eingliederungsmodell hat in Deutschland die größte Prominenz erlangt - eine erfolgreiche Eingliederung nur über den Weg einer Assimilation gelingen kann, ${ }^{3}$ weisen andere Autoren auf Vorteile eigenethnischer Ressourcen bei diesem Prozess hin. ${ }^{4}$ Vor allem bei stark benachteiligten Gruppen, bei denen eine Assimilation aufgrund ihrer sozioökonomischen Ausgangssituation und ihres Wohnkontextes nicht mit Erfolg verknüpft sein muss, ${ }^{5}$ können herkunftslandspezifische Werte und Normen sowie Netzwerke zur Ressource werden. Dies ist gemeinhin unter dem Begriff Sozialkapital zu verstehen. Religiöse Werte und Netzwerke sind besonders dafür prädestiniert, Sozialkapital zu entwickeln. ${ }^{6}$ So ist anzunehmen, dass fromme Muslime von den mit ihrer Religiosität verknüpften Ressourcen auf dem Weg zu einer erfolgreichen Eingliederung profitieren können. $\mathrm{Zu}$ berücksichtigen ist, dass sich die religiöse Orientierung auch unter frommen Muslimen stark unterscheiden kann und zudem durch Lebensumstände Wandlungen unterliegt. Unterschiedliche Zugänge zum Islam können mit Unterschieden in der Sozialkapitalaustattung und -struktur einhergehen.

In diesem Beitrag liegt der Fokus auf zwei muslimischen Gruppen, die deutliche Unterschiede in ihrem Sozialkapital aufweisen. Zum einen geht es um Muslime mit einem reformistischen Zugang zum Islam (im Folgenden auch Neo-Reformisten oder Neo-Orthodoxe genannt). Diese zeichnen sich durch Frömmigkeit und gleichzeitig durch eine zeitgemäße Auslegung der religiösen Quellen aus. Zum anderen sind Muslime mit einem klassisch orthodoxen Zugang zum Islam von Interesse. Diese halten an einer wortgetreuen Lesart der religiösen Primärquellen fest. Während Anhänger des Reformislam eher gruppenübergreifendes Brücken bildendes Sozialkapital (sog. bridging social capital) anstreben bzw. bereits darüber verfügen, so die Annahme, ist für Anhänger des orthodoxen Islam vornehmlich gruppeninternes bindendes Sozialkapital (sog. bonding social

\footnotetext{
${ }^{3}$ Vgl. Hartmut Esser 2001.

${ }^{4}$ Vgl. Alejandro Portes und Min Zhou 1993 sowie Min Zhou 1997.

${ }^{5}$ Nach Alejandro Portes und Ming Zhou (1993) führt die Assimilation an das „falsche“ Segment der Gesellschaft zur downward assimilation bzw. zum dauerhaften Verbleib in der Unterschicht. Eine Assimilation an das falsche Gesellschaftssegment geht mit der Übernahme von unerwünschten Werten und Normen einher und zeigt sich in devianten Verhaltensweisen. Je nach Wohnkontext kann es passieren, dass keine Kontaktmöglichkeit zu Angehörigen der Mittelschicht - die meist als Vorbild für Assimilationsmodelle fungieren - besteht.

${ }^{6}$ Vgl. Robert Putnam 2000.
} 
capital) von Bedeutung. Entsprechend unterscheiden sich die Integrationswege und -vorstellungen beider Gruppen.

Anhand von Fallbeispielen werden Unterschiede in der Sozialkapitalausstattung beider genannten religiösen Lager aufgezeigt und mögliche Erklärungen dafür dargestellt. Es wird auf die Organisations- und Mitgliedsstruktur, die Vernetzungen mit anderen Gruppen und Organisationen sowie das soziale Engagement ausgewählter muslimischer Organisationen bzw. Vereine in Deutschland eingegangen.

\section{Strömungen im gegenwärtigen sunnitischen Islam}

Es ist schwierig, die verschiedenen Strömungen im Islam fassbar zu machen, weil zumindest der sunnitische Islam, dem die überwiegende Mehrheit aller Muslime zugeordnet werden kann, keine institutionalisierte Religion ist und es deshalb sehr stark von Lehrmeinungen und Deutungen abhängt, welche konkrete Ausprägung er annimmt. Ein zentrales Unterscheidungskriterium ist der Umgang mit den religiösen Primärquellen - dem Koran und der Sunna (gesammelte Überlieferungen über die Aussprüche und Taten des Propheten Muhammad). Auf diese Weise kann zwischen vier Hauptströmungen unterschieden werden: ${ }^{7}$ dem ethischen Islam, dem skripturalistischen Islam, dem traditionellen Islam und der islamischen Mystik. Allerdings ist zu beachten, dass es sich hierbei nicht um trennscharfe Kategorien handelt. Empirisch gibt es zahlreiche Überschneidungen, und verschiedene religiöse Gruppen lassen sich meist nicht eindeutig der einen oder anderen Strömung zuordnen.

Der ethische Islam ist mit einer privatisierten Religiosität nach unserem eher säkular-humanistischen Verständnis vergleichbar. Die Primärquellen bzw. der Koran wird eher als Buch, das ethische Richtlinien enthält, angesehen. Für die alltägliche Lebensgestaltung spielt er eine eher geringe Rolle.

Der skripturalistische Islam zeichnet sich dadurch aus, dass er nur die Primärquellen anerkennt, d. h. den Koran und die Sunna - in vielen Fällen sogar nur den Koran. Und diese spielen für die Lebensgestaltung eine zentrale Rolle, weil sie Richtlinien enthalten, wie ein Leben nach islamischen Vorschriften auszuse-

\footnotetext{
${ }^{7}$ Eigene Systematisierung verschiedener empirischer und theoretischer Typologien. Vgl. auch Tariq Ramadan 2001, S. 302.
} 
hen hat. Volksislamische Praktiken werden strikt abgelehnt. Die verschiedenen Rechtsschulen, die im Laufe der Zeit entstanden sind und eigene Auslegungstechniken entwickelt haben, spielen eine eher untergeordnete Rolle. Innerhalb dieser Strömung lassen sich verschiedene Unterformen unterscheiden, die weiter unten beschrieben werden.

Der traditionelle Islam bezieht sich auch auf die Primärquellen, aber im Unterschied zum skripturalistischen Islam finden auch volksislamische Praktiken Platz darin. Hierbei handelt es sich meist um lokale Ausformungen des Islam, wie sie im Herkunftsland lange tradiert und von der ersten Migrantengeneration nach Deutschland mitgebracht wurden. Die Praktiken unterscheiden sich graduell je nach lokaler Prägung. Die Auslegung der Primärquellen durch die jeweiligen Rechtsschulen ist für diese Strömung von Bedeutung. Dabei gibt es eine Bandbreite zwischen eher nicht so strengen Auslegungen, wie z. B. bei den Hanafiten, und strengeren Auslegungen, wie z. B. bei den Hanbaliten.

$\mathrm{Zu}$ erwähnen ist natürlich auch die islamische Mystik - der Sufismus -, der einen spirituellen, keinen kognitiven Zugang zum Koran sucht. Hier herrscht meist die Ansicht, dass der Inhalt des Korans nur über Meditation und die so erreichte Nähe zu Gott erschlossen werden kann. Die meisten lokalen Ausprägungen des Islam, der so genannte „Volksislam“, war immer eine Mischung von Traditionalismus und Mystik, wird aber heute immer stärker vom skripturalistischen Islam verdrängt bzw. als unislamisch abgewertet. Entsprechend verliert der Volksislam zunehmend an Bedeutung, und rational-skripturalistisch orientierte Formen gewinnen an Einfluss.

Wenn es um Re-Vitalisierung des Islam geht, handelt es sich meist um den skipturalistischen Islam. Dieser ist jedoch keineswegs homogen. Zunächst ist zwischen orthodoxen und reformistischen Skripturalisten zu unterscheiden. Die Orthodoxen zeichnen sich durch eine wortgetreue Lesart des Korans aus und lehnen die Interpretation der Inhalte ab. Dies kann auch als klassische Orthodoxie bezeichnet werden. Für die Reformisten bzw. reformistischen Skripturalisten ist die zeitgemäße Interpretation der Quellen zentral. Allerdings unterteilen sich die Reformer in zwei Lager: Die einen, die Neo-Reformer, sind der Ansicht, dass Moderne und Islam durchaus miteinander kompatibel seien und auch moderne Staatsformen kein Problem darstellen; der Islam müsse an die neuen Umstände 
angepasst werden bzw. moderne Werte seien durch den Koran begründbar. Werner Schiffauer bezeichnet diese Gruppe als Neoorthodoxe oder Postislamisten. Seiner Definition nach

[...] setzt sich [die Neo-Orthodoxie] von der klassischen Orthodoxie ab, insofern sie einen ictihad, eine Weiterentwicklung der Rechtslehre fordert. Sie setzt sich andererseits von einem ,Europäischen Islam' ab, wie er immer von europäischen Journalisten und Politikern gefordert wird, insofern sie dem Prinzip der Recht-Leitung, also der Ausrichtung der Lebensführung am göttlichen Willen, wie er Muhammad offenbart wurde, festhält. ${ }^{8}$

Die anderen, die Neo-Islamisten bzw. Neo-Fundamentalisten, ${ }^{9}$ lesen dagegen den Koran zwar auch dynamisch, aber sehr selektiv, weil sie ein konkretes Ziel vor Augen haben: Das politische Handeln steht hier im Zentrum sowie das Ziel der Errichtung eines islamischen Gottesstaates. Dabei gibt es solche, die Gewalt vehement ablehnen und eher die Macht der Worte und Argumente vorziehen, während die anderen durchaus als gewaltbereit einzustufen sind.

Der Fokus des Beitrages liegt zum einen auf Gruppen, die der klassischen Orthodoxie zuzuordnen sind, und zum anderen auf Gruppen, die als Neo-Reformisten bzw. Neo-Orthodoxe bezeichnet werden können. ${ }^{10}$ Ein weiteres Unterscheidungsmerkmal beider Gruppen ist, dass Anhänger der klassischen Orthodoxie meist Angehörige der ersten Migrantengeneration sind, Neo-Reformisten bzw. Neo-Orthodoxe sind meist Angehörige der zweiten und dritten Migrantengeneration. Letztere sind nicht selten in einem klassisch orthodoxen Milieu aufgewachsen, haben aber aufgrund ihrer Sozialisation in der hiesigen Gesellschaft eine neo-reformistische bzw. neo-orthodoxe Orientierung entwickelt. ${ }^{11}$

\section{Sozialkapital frommer Muslime}

In unterschiedlichen theoretischen Ansätzen werden übereinstimmend vier zentrale Elemente von Sozialkapital genannt. Diese sind soziale Beziehungen, Ressourcen, Normen und Vertrauen. Nach Putnam kann Sozialkapital wie folgt definiert werden: „By ,social capital', I mean features of social life - networks,

\footnotetext{
${ }^{8}$ Werner Schiffauer 2010, S. 23.

${ }^{9}$ Vgl. Olivier Roy 2006.

${ }^{10}$ Vgl. Werner Schiffauer 2010.

${ }^{11}$ Vgl. Werner Schiffauer 2012, S. 285-287.
} 
norms, and trust - that enable participants to act together more effectively to pursue shared goals ". ${ }^{12}$ In Beziehungsnetzwerken werden Putnam zufolge durch Kooperation der Mitglieder Reziprozitätsnormen generiert, welche die Kooperation untereinander erleichtern. Vor allem Religionsgemeinschaften seien besonders prädestiniert für die Produktion von Sozialkapital; Solidarität unter den Mitgliedern sei bereits durch ein Zugehörigkeitsgefühl zur Gruppe vorhanden. Normen und Vertrauen unter den Mitgliedern müsse nicht erst hergestellt werden, sondern werde bereits durch religiös vermittelte Werte bereitgestellt. ${ }^{13}$ Nach Lukka ist „religiöses Kapital“ eine Unterform von Sozialkapital.

Zentral für die Stabilität der Gruppe sind insbesondere „offene Rechnungen“. Diese ergeben sich vor allem dann, wenn die Gegenleistung nicht direkt vom Empfänger erbracht wird, sondern z. B. durch die Gruppe insgesamt in Form von Anerkennung und Prestige. Vor allem soziales Engagement fördert das Prestige eines Akteurs in der Gruppe oder einer Gruppe in der Gesellschaft. Damit kommt gesellschaftlichem Engagement eine wichtige Funktion zu: Es fördert das Vertrauen in diese Gruppe und deren Ansehen. Soziales Engagement führt entsprechend zur Produktion von symbolischem Kapital, ${ }^{14}$ was zentral ist für die Erlangung von Macht in der Gesellschaft und somit für die Durchsetzung von Interessen. Diesem Aspekt kommt in Bezug auf muslimische Gruppen in Deutschland eine zentrale Bedeutung zu, der weiter unten näher erläutert wird. Eine weitere wichtige Dimension des Sozialkapitals für das Verständnis der Integrationsbemühungen von muslimischen Gruppen in Deutschland ist die Unterscheidung zwischen Brücken bildendem und bindendem Sozialkapital.

Brückenbildendes Sozialkapital bezieht sich auf soziale Netzwerke, die völlig unterschiedliche Menschen zusammenbringen; bindendes Sozialkapital bringt in einigen Punkten (wie Ethnizität, Alter, Geschlecht, soziale Klasse usw.) ähnliche Menschen zusammen. [...] In der Praxis sind allerdings die meisten Gruppen brückenbildend und bindend zugleich. ${ }^{15}$

Das trifft auch auf muslimische Gemeinschaften in Deutschland zu. Moscheevereine wurden schon zu Zeiten der so genannten Gastarbeiterwelle in den

\footnotetext{
${ }^{12}$ Robert D. Putnam 1995, S. $664 \mathrm{ff}$.

${ }^{13}$ Vgl. Priya Lukka 2003.

${ }^{14}$ Vgl. Pierre Bourdieu 1983.

${ }^{15}$ Robert D. Putnam 2001, S. 28 ff.
} 
1970er Jahren gegründet, dienten aber nicht nur als Gebetsstätte, sondern stellten ein wichtiges Bindeglied in die Heimat dar. ${ }^{16}$ Die Solidarität untereinander wurde gestärkt; die frühen Moscheevereine hatten vor allem bindenden Charakter - nicht nur konfessionell, sondern insbesondere auch ethnisch. Werte und Normen der Herkunftsgesellschaft erfuhren hier eine gewisse Form der Institutionalisierung in der „Fremde“. Darüber hinaus entwickelten sich Moscheevereine zu einer wichtigen Anlaufstelle für die Probleme vieler Migranten aus demselben Land - die besser gebildeten unter ihnen boten ihre Unterstützung an, beispielsweise bei Behördengängen oder beim Ausfüllen von Formularen. Vor allen Dingen für Neuankömmlinge waren sie eine wichtige Anlaufstelle. Entsprechend stellten und stellen sie auch heute noch eine zentrale soziale Ressource in der muslimischen Migrantencommunity dar. Der Mangel an ökonomischen und kulturellen Ressourcen wurde so durch soziale Ressourcen ausgeglichen.

Diese Herkunftslandorientierung löst sich in einigen Vereinen zunehmend auf bzw. wird zunehmend unwichtiger. Es werden nicht nur Muslime unterschiedlicher Herkunft in Deutschland stärker einbezogen, sondern auch zunehmend Brücken in andere muslimische Länder weltweit gebaut. Brücken innerhalb Deutschlands, zu deutschen Institutionen und Organisationen, hat es zwar schon immer gegeben, werden aber immer stärker ausgebaut. Es sind gar neue religiöse Vereine und Netzwerke auszumachen - vor allem bestehend aus Mitgliedern der zweiten oder dritten Migrantengeneration -, deren zentrale Ausrichtung die Brückenbildung in die deutsche Gesellschaft darstellt.

Es können zwei Tendenzen in der muslimischen Vereinslandschaft ausgemacht werden, die jeweils - so die These - Wege aus der starken Begrenzung auf die ethnische Kolonie darstellen. Es kann angenommen werden, dass es sich in beiden Fällen um Strategien handelt, die engen Grenzen der Mobilität in der ethnischen Kolonie zu überwinden und langfristig die Position der Muslime in Deutschland zu verbessern, allerdings ohne sich vollständig von ihr abzunabeln. ${ }^{17}$ Bereits Putnam betont, dass eine stark bindende Ausrichtung eines Ver-

\footnotetext{
${ }^{16}$ Vgl. den Beitrag von Martina Sauer und Dirk Halm (S. 389-417) in diesem Sammelband.

${ }^{17}$ Nach Hartmut Esser (2000) ist der soziale Aufstieg nur durch Assimilation an die Aufnahmegesellschaft möglich - dafür sei aber das Verlassen des ethnischen Schutzraumes (Binnenintegration)
} 
eins - so wie die frühen Moscheevereine in der ersten Phase - eine notwendige Voraussetzung für eine spätere Brücken bildende Orientierung ist.

Im Folgenden werden zwei grundsätzlich voneinander zu unterscheidende muslimische Gruppen betrachtet, die ihre Brücken in unterschiedliche Richtungen ausbauen. Während die einen vor allen Dingen Brücken bildendes Sozialkapital in die Aufnahmegesellschaft - also in Deutschland - anstreben, kooperieren die anderen verstärkt mit Muslimen bzw. muslimischen Organisationen weltweit, besonders im Nahen Osten. Entsprechend kann der zweite Typ muslimischer Organisationen im Hinblick auf die konfessionelle Ausrichtung der Netzwerke als bindend bezeichnet werden, da sie sich speziell auf Muslime konzentrieren, während erstere vor allem Kontakte in die hiesige Gesellschaft und somit zu nicht-muslimischen Institutionen und Organisationen anstreben und daher konfessionell eine überwiegend Brücken bildende Ausrichtung aufweisen.

Die These ist, dass sich in Abhängigkeit von der Kapitalsorte bzw. von der Ressource, auf die jeweils gesetzt wird, die Strategien, um die Position der Muslime in der Gesellschaft zu verbessern, stark unterscheiden. Auch wenn Muslime in Deutschland noch immer über ein vergleichsweise geringeres Bildungsniveau und über weniger ökonomisches Kapital verfügen, wurde über die Jahre relativ viel von beiden Kapitalien angehäuft. Der Anteil mit Abitur und Universitätsabschluss hat bei der zweiten und dritten Generation deutlich zugenommen, ${ }^{18}$ und es wurde relativ viel Geld angespart. Religiöses Sozialkapital, so die These, verspricht nun gute Erfolge bei der „gewinnbringenden“ Umwandlung dieser Ressourcen.

Um kulturelles Kapital, also Bildungskapital, auch entsprechend anzuwenden, ist es erforderlich, in das Positionssystem der hiesigen Gesellschaft einzudringen. Entsprechend ist Brücken bildendes Sozialkapital in Deutschland notwendig. Um das Prestige der eigenen Gruppe in Deutschland zu erhöhen, ist gerade soziales Engagement in Deutschland zentral.

notwendig. Der Verbleib in der ethnischen Gemeinde gehe mit begrenzten Aufstiegsmöglichkeiten einher (Mobilitätsfalle). Siehe auch Norbert F. Wiley 1967.

${ }^{18}$ Vgl. Martina Sauer und Dirk Halm 2009. 
Ökonomisches Kapital kann gewinnbringend bzw. die Position der Gruppe stärkend eingesetzt werden, wenn eigene Märkte entworfen werden können. Religiöse Normen bzw. religiöses Sozialkapital kann hier einen spezifischen Markt generieren (bspw. Halal-Produkte) und gleichzeitig den wirtschaftlichen Erfolg garantieren, wenn diese religiösen Normen von einer wachsenden Anzahl von Muslimen befolgt werden. Dafür ist eher bindendes Sozialkapital - also verstärkt Sozialkapital mit Muslimen - erforderlich, allerdings weltweit. Indem das soziale Engagement nicht auf eine kleine Gruppe beschränkt, sondern auf Muslime weltweit erweitert wird, können auch die spezifischen religiösen Deutungen bzw. Werte und Normen, die diesen spezifischen Markt tragen, exportiert werden.

Weiterhin wird angenommen, dass diese unterschiedlichen Ausrichtungen bezüglich des Sozialkapitals mit unterschiedlichen religiösen Orientierungen der entsprechenden Gruppen einhergehen. Während muslimische Gruppen mit einer transnational, aber konfessionell bindenden Sozialkapitalausrichtung eher eine klassische orthodoxe religiöse Ausrichtung aufweisen, sind diejenigen, die nationales, aber interkonfessionelles Brücken bildendes Sozialkapital anstreben, eher den Neo-Reformisten bzw. Neo-Orthodoxen mit einer relativ liberalen Deutung des Islam zuzuordnen.

Im Folgenden wird auf beide Vereinstypen näher eingegangen und exemplarisch anhand jeweils eines islamischen Vereins in Deutschland beschrieben. Dabei wird auf die Vereinsstruktur, auf spezifisch religiöse Werte und Normen, ihre Netzwerke und schließlich das soziale Engagement der Gruppen als zentrale Aspekte von Sozialkapital eingegangen. ${ }^{19}$

\subsection{Klassische Orthodoxie und Sozialkapital}

Merkmal der klassisch-orthodoxen Orientierung im Islam ist eine buchstabengetreue Lesart der religiösen Primärquellen - die Interpretation bzw. Abwandlung religiöser Gebote wird nicht akzeptiert. Die Orientierung an diesen Normen und Werten schränkt die Nutzung gängiger Märkte und der dort angebotenen Produkte ein und erfordert neue Märkte und Produkte, die diesen spezifischen Be-

\footnotetext{
${ }^{19}$ Die Informationen wurden dem Internetauftritt der Vereine und zum Teil aus der Vereinszeitschrift entnommen.
} 
darf bedienen (Halal-Produkte und weitere islamkonforme Produkte). So hat der rationale Zugang zum Islam, in diesem Fall eine rigide Auslegung der religiösen Primärquellen, zum Wirtschaftsaufschwung in der islamischen Welt geführt, die ohne diese „neue Orthodoxie“ nicht denkbar gewesen wäre. Dies soll am Beispiel ,scharia-konformer' Finanzprodukte erläutert werden. Nach islamischem Recht sind Zinsen, Wucher und die Investition in Branchen, die mit Alkohol, Glücksspiel, Tabak und Schweinefleisch handeln, verboten. Entsprechend konnten sich islamische Finanzprodukte weltweit etablieren und wachsen jedes Jahr um 20 bis 30 Prozent $^{20}$. Islamic-bancing ist mittlerweile einer der am schnellsten wachsenden Sektoren in der globalen Finanzdienstleistungsindustrie. Jede Bank, die islamische Finanzprodukte anbietet, benötigt ein so genanntes „Sharia-board“, das sich aus Religionsgelehrten zusammensetzt und entscheidet, welche Finanzprodukte tatsächlich islamkonform sind und welche nicht. Das bedeutet auch eine Verwertbarkeit des spezifisch religiösen Kulturkapitals. In Deutschland bieten mittlerweile auch einige Banken islamische Finanzprodukte an und lassen sich z. B. vom Zentralrat der Muslime diesbezüglich beraten. Religiöse Gruppen bzw. Organisationen, die der klassischen Orthodoxie zugeordnet werden können, streben insbesondere auch transnationales Sozialkapital unter Muslimen an und stärken das Vertrauen untereinander. Viele islamische Gruppen sind mittlerweile transnational mit anderen muslimischen Gruppen vernetzt oder haben ihren Verein weltweit ausgebaut. Das soziale Engagement erstreckt sich auf die gesamte islamische Welt, beschränkt sich aber auf Muslime. Bisher waren islamische Orientierungen sehr kontextspezifisch und eher auf kleinere lokale Einheiten beschränkt. Transnationale Vernetzungen führen zum weltweiten Export von Ideen und Deutungen und unterstützen damit nicht nur die Globalisierung spezifischer religiöser Orientierungen, sondern auch der islamischen Ökonomie. Damit werden langfristig weltweit - auch in Deutschland - spezifische ökonomische Strukturen und ein entsprechendes Positionssystem geschaffen, dass die Position der Muslime in der Gesellschaft stärkt.

Die Islamische Gemeinschaft Milli Görüs (IGMG) kann als ein Beispiel für einen religiösen Verein mit transnationalen Vernetzungen angeführt werden, der vor allem bindendes Sozialkapital produziert und der klassischen Orthodo-

\footnotetext{
${ }^{20}$ Vgl. Friedrich Thießen und Mathias Saggau 2009.
} 
xie zugeordnet werden kann. Die IGMG sieht die „religiöse Wegweisung“ als ihre zentrale Aufgabe. In einem Absatz aus ihrer Selbstbeschreibung wird ihr Islamverständnis wie folgt zusammengefasst:

Grundlage des Islamverständnisses der IGMG sind die Lehren von Koran und Sunna. Beide Quellen sind richtungweisend sowohl für die Gemeinschaft als auch für ihre Mitglieder. Der Islam ist im gesellschaftlichen und individuellen Bereich eine Lebensweise, deren Einfluss nicht an der Moscheetür endet, sondern auch im Alltagsleben der Muslime mit moralisch-ethischen Werten und Vorgaben eine maßgebliche Rolle spielt. Hierbei geht es der IGMG nicht um die Konservierung bestimmter regionalkultureller Formen der Religiosität, sondern um die Auslebung des Glaubens im Lichte des Korans und der darauf aufbauenden Tradition des Propheten. ${ }^{21}$

Ein so genannter Fiqh-Rat ist für die Auslegung der Lehre zuständig. Die transnationale Orientierung des Vereins wird im folgenden Zitat explizit bekundet: „Die IGMG bekennt sich auch zu ihrer Verantwortung gegenüber der weltweiten muslimischen Gemeinschaft (Umma) und setzt sich für die Lösung der Probleme der Umma ein." Der Verein ist hierarchisch organisiert: Den Moscheegemeinden sind Regionalverbände übergeordnet, denen wiederum der Europaverband übersteht. Religiöse Dienstleistungen, die Bildungsarbeit und soziale Angebote werden zentralistisch koordiniert. In Deutschland verfügt die IGMG nach eigenen Angaben über 323 Moscheegemeinden - hinzu kommen diverse Frauen-, Jugend-, Sport- und Bildungsvereine. Nach eigenen Angaben liegt die Zahl der eingetragenen Mitglieder bei 87.000 - die Gemeindegröße bei etwa 300.000 (,Freitagsgemeinde').

Offiziell finanziert sich die IGMG nur durch Spenden. Jedes Mitglied zahlt einen monatlichen Beitrag von etwa zehn bis 30 Euro. Zusätzlich ist nach dem religiösen Gebot des zakat jährlich ein festgelegter Anteil des Vermögens zu spenden (2,5 Prozent) sowie zum Ende des Fastenmonats Ramadan vor dem Gemeindegebet ein fester Betrag von neun Euro zu entrichten (fitra). Es ist bekannt, dass die IGMG über einflussreiche Kontakte im Nahen Osten verfügt und auch größere Spenden aus dieser Region erhält. Die durch Spenden gesammelten Gelder werden für soziale Zwecke eingesetzt - das soziale Engagement beschränkt sich auf Muslime weltweit in benachteiligten oder von Katastrophen betroffenen Regionen. Darüber hinaus werden Stipendien für das Studium an der Al-

${ }^{21}$ Unter: http://www.igmg.de (07.09.2012). 
Azhar Universität in Kairo vergeben. Soziale Tätigkeiten im Jahr 2008 waren u. a.: Verteilung von Lebensmittelpaketen an 3.300 bedürftige Familien im Osten der Türkei, Hilfe auf dem Gaza-Streifen: in Zusammenarbeit mit der Union Arabischer Mediziner wurde die bedürftige Bevölkerung mit Nahrungs- und Arzneimitteln versorgt; Schwerverletzte wurden bzw. werden in Istanbuler und Kairoer Krankenhäusern versorgt; Bangladesch: Verteilung von Lebensmittelpaketen sowie Kleidung und Decken nach Super-Zyklon, Errichtung von Waisenhäusern, Krankenhäusern und Gebetsstätten (langfristig) in verschiedenen Städten, Baugrundstück zum Teil von wohlhabenden Einheimischen gespendet, Fertigstellung war Ende 2008 geplant.

\subsection{Reformislam bzw. Neo-Orthodoxie und Sozialkapital}

Anhänger des Reformislam bzw. der Neo-Orthodoxie können als ebenso fromm bezeichnet werden wie Anhänger der islamischen Orthodoxie (rituelle Pflichten sind ebenso wichtig), allerdings weichen sie zum Teil in der Lesart des Korans bezüglich gesellschaftlicher Aspekte von der Orthodoxie ab. Sie betonen explizit, dass eine dynamische und zeitgemäße Interpretation der religiösen Quellen erforderlich ist und jeder Einzelne selbst die Verantwortung dafür trägt. Jeder Einzelne habe die Aufgabe, sich um eine adäquate Auslegung der religiösen Quellen zu bemühen und sei dafür entsprechend mit Verstand ausgestattet. Dabei berufen sie sich auf Sure 96, die mit dem Wort „lies! (iqra)“ beginnt. Wichtig ist dieser Gruppe, zu zeigen, dass sich moderne Werte (wie z. B. Gleichberechtigung von Mann und Frau) und Islam nicht ausschließen, sondern diese sogar durch den Islam selbst begründet werden können. Dies versuchen sie durch entsprechende Stellen im Koran oder der Sunna zu belegen.

Die oben genannte Sure 96 ist insgesamt zentral für diese Bewegung. Aus ihr wird abgeleitet, dass Bildung und Wissen religiöse Werte an sich seien. Es sei als religiöse Pflicht zu verstehen, sich zu bilden und zu forschen. Entsprechend handelt es sich bei den Mitgliedern durchweg um hoch gebildete Gläubige. Sie haben meist die hiesigen Bildungseinrichtungen durchlaufen und wollen nun auch entsprechende Positionen im hiesigen Positionssystem besetzen. Um dies zu erreichen, so die Annahme, streben sie vor allem Brücken bildendes Sozialkapital an. Dies wird auch an ihren Bemühungen ersichtlich: Es wird versucht, das Image 
des Islam in der Gesellschaft zu verbessern. Dafür ist der Aufbau von Vertrauen (Kreditwürdigkeit) in der Gesellschaft erforderlich, das sie durch gemeinnützige Tätigkeiten, die vorwiegend auch der hiesigen Gesellschaft und nicht nur Muslimen zu Gute kommen, anstreben. Des Weiteren versuchen sie Netzwerke mit zentralen Institutionen der Gesellschaft aufzubauen. Dafür werden Diskussionsabende organisiert, zu denen namhafte Politiker eingeladen werden. Es finden regelmäßige interkulturelle Veranstaltungen statt. Ziel ist es, aktiv durch bürgerschaftliches Engagement an der Gesellschaft zu partizipieren bzw. diese mitzugestalten und durch Sichtbarkeit positiv auf das Bild des Islam in der Gesellschaft einzuwirken. Sie empfinden sich als Teil der Gesellschaft und wollen auch als solcher anerkannt werden.

Die Muslimische Jugend Deutschland (MJD) kann dem Reformislam zugeordnet werden und strebt ihren Aktivitäten zufolge vor allem Brücken bildendes Sozialkapital an. Der Selbstdarstellung zufolge ist es ihr Ziel, den interreligiösen Dialog voranzutreiben sowie Vorurteile über den Islam in der Gesellschaft abzubauen und muslimischen Jugendlichen, die sich in der Schule oder im Beruf Diskriminierung ausgesetzt sehen, zu mehr Selbstbewusstsein zu verhelfen. $\mathrm{Zu}-$ dem unterstützen sie muslimische Jugendliche mit Integrationsproblemen und veranstalten Aufklärungskampagnen gegen Jugendgewalt und Kriminalität bzw. bieten Freizeitaktivitäten an. Der Verein ist netzwerkartig organisiert und besteht aus verschiedenen unabhängigen Lokalkreisen. Einmal im Jahr wird im Rahmen einer Mitgliederversammlung der Vorstand gewählt. Die Mitglieder entrichten einen Mitgliedsbeitrag von drei Euro im Monat und können sich in verschiedenen AGs sozial engagieren. Ehrenamtliches Engagement ist zentral. Die MJD finanziert sich hauptsächlich durch den Mitgliedsbeitrag sowie Spenden und durch Projektförderungen von staatlicher Seite. Zu den ehrenamtlichen Tätigkeiten zählen neben der Unterstützung von Jugendlichen bei Integrationsproblemen z. B. soziales Engagement beim Hochwasser in Sachsen im Jahre 2002, das Verteilen von Lunch-Paketen an Obdachlose wie auch das Verteilen von Süßspeisen in Krankenhäusern und anderen sozialen Einrichtungen zum Zuckerfest. Zudem organisiert die MJD interreligiöse Seminare zu verschiedenen gesellschaftlichen Themen (z.B. Demokratie und Toleranz) zusammen mit Jugendorganisationen anderer Religionsgemeinschaften und Initiativen z. B. 
gegen Zwangsheirat. Des Weiteren ist die MJD an Projekten beteiligt, die von Ministerien finanziell gefördert werden. Im Jahre 2003 gab es Gelder vom Bundesfamilienministerium für ein Projekt gegen Gewalt. Im Frühjahr 2008 organisierte die MJD einen Diskussionsabend zum Thema "Jugendgewalt", an dem namhafte Politiker verschiedener Parteien in Deutschland teilnahmen. Gegenwärtig handelt es sich bei der MJD um ein breit ausgebautes Netzwerk, dass mit verschiedenen Organisationen und Vereinen der hiesigen Gesellschaft vernetzt ist.

Neben der MJD ist auch die Jugendorganisation der Milli Görüs dieser Gruppe zuzurechnen. Schiffauer zufolge konzentriert sich die neue Generation der IGMG stärker auf Deutschland und bemüht sich um Vernetzungen mit der hiesigen Gesellschaft. Zunehmend übernimmt diese neue Generation - es handelt sich auch hier um Angehörige der zweiten und dritten Migrantengeneration - Führungspositionen bei der IGMG. Mittlerweile wird etwa jeder 10. IGMGVerein von Angehörigen der Nachfolgegeneration geführt. Merkmale der neuen Führungsriege der IGMG sind, dass sie sich in Deutschland zuhause fühlen, die hiesigen Bildungsinstitutionen durchlaufen haben und Teil der Gesellschaft werden wollen - ähnlich wie Angehörige der MJD. ${ }^{22}$ Es ist davon auszugehen, dass sie den Kurs der IGMG mit der Zeit zunehmend in eine andere Richtung lenken werden und auch hier stärker brückenbildendes Sozialkapital an Bedeutung gewinnen wird.

\section{Schlussbetrachtung}

Ziel des Beitrages war es zu zeigen, dass sich fromme Muslime - entgegen der häufig vertretenen Annahme, der Islam stelle generell ein Integrationshindernis dar - ihre eigenen Integrationswege in die deutsche Gesellschaft suchen. Die religiöse Organisation bzw. das mit der religiösen Einbindung zur Verfügung stehende Sozialkapital wirkt dabei unterstützend und nimmt eine zentrale Rolle für das weitere Vorgehen ein. Maßgeblich zwei islamische Gruppen sind in dieser Hinsicht interessant, fast exemplarisch, die sich sowohl im Hinblick auf ihre religiöse Orientierung als auch bezüglich ihrer Sozialkapitalausstattung beträchtlich unterscheiden. Während Anhänger des Reformislam bzw. der Neo-Or-

\footnotetext{
${ }^{22}$ Vgl. Werner Schiffauer 2010.
} 
thodoxie eher gruppenübergreifendes Brücken bildendes Sozialkapital anstreben bzw. bereits darüber verfügen, ist für Anhänger des klassisch orthodoxen Islam vornehmlich gruppeninternes bindendes Sozialkapital von Bedeutung. Entsprechend unterscheiden sich auch die Integrationswege und Vorstellungen beider Gruppen. Während die ersteren versuchen, durch einflussreiche Kontakte in die hiesige Gesellschaft und gemeinnützige Tätigkeiten, die nicht nur Muslimen, sondern auch Nicht-Muslimen zugutekommen, Vertrauen und Akzeptanz in der deutschen Gesellschaft zu erlangen, konzentrieren sich die letzteren auf gruppeninterne Kontakte weltweit, um ihren Einfluss auszubauen.

Als eine Erklärung für die unterschiedlichen Strategien der beiden Gruppen kann angeführt werden, dass beide Gruppen jeweils auf unterschiedliche Ressourcen setzen: Während sich Anhänger des Reformislam eher auf die Umwandlung von kulturellem Kapital konzentrieren bzw. auf die Übernahme von angesehenen Positionen in der deutschen Gesellschaft - entsprechend zu ihren hier erworbenen relativ hohen Bildungsabschlüssen -, setzt die klassische Orthodoxie vornehmlich auf ökonomisches Kapital. Die „konservative“ Auslegung der religiösen Normen hat einen spezifisch islamischen Markt generiert, der sich durch den Export dieser Normen auch weltweit etabliert hat und zunehmend wächst, wie am Beispiel des Islamic Banking gezeigt werden konnte.

Abschließend kann gesagt werden, dass es sich in beiden Fällen um rationale und langfristig Erfolg versprechende Integrationsbemühungen handelt, ohne sich von der schützenden Migrantencommunity direkt abnabeln zu müssen. Die dort vorhandenen Ressourcen werden genutzt, um aus ihr herauszutreten bzw. die bisher in sich geschlossenen Communitys stärker mit der deutschen Gesamtgesellschaft bzw. transnational zu verweben. Interessant ist weiterhin, dass Anhänger der klassischen Orthodoxie meist Angehörige der ersten Migrantengeneration sind, während sich Neo-Reformisten bzw. Neo-Orthodoxe aus Angehörigen der Nachfolgegeneration zusammensetzen. ${ }^{23}$ Es kann davon ausgegangen werden, dass es sich um zeitlich versetzte Entwicklungen handelt, wie sich auch aus dem internen Wandel der IGMG - ihre religiöse wie auch gesellschaftliche Ausrichtung betreffend - ablesen lässt: Das konservative Milieu der Elterngeneration und die darin bereitgestellten Ressourcen (Sozialkapital) haben zum

\footnotetext{
${ }^{23}$ Vgl. ebenda.
} 
relativen Bildungserfolg der Nachfolgegenerationen beigetragen. Diese stoßen nun jedoch auf gesellschaftliche Barrieren, die sie daran hindern, ihre Bildung in entsprechende Positionen in der Gesellschaft zu übersetzen. ${ }^{24}$ Die neo-orthodoxe religiöse Ausrichtung sowie das Streben nach brückenbildendem Sozialkapital der jungen Muslime können als Streben nach Anerkennung verstanden werden. Vielleicht entwickeln sich auf diese Weise die so genannten ethnischen Kolonien letzten Endes doch von Fallen ${ }^{25}$ zu Schleusen ${ }^{26}$.

\section{Literatur}

Aumüller, Jutta, Assimilation. Kontroversen um ein migrationspolitisches Konzept. Bielefeld 2009.

Bourdieu, Pierre, Ökonomisches Kapital, kulturelles Kapital, soziales Kapital, in: Reinhard Kreckel (Hrsg.), Soziale Ungleichheiten, Soziale Welt, Sonderheft 2: Göttingen 1983, S. 183-198.

Brettfeld, Katrin und Wetzels, Peter, Muslime in Deutschland. Integration, Integrationsbarrieren, Religion und Einstellungen zu Demokratie, Rechtsstaat und politisch-religiös motivierter Gewalt. Ergebnisse von Befragungen im Rahmen einer multizentrischen Studie in städtischen Lebensräumen. Im Auftrag des Bundesministeriums des Innern, Berlin 2007.

Elwert, Georg, Gesellschaftliche Integration durch Binnenintegration?, in: Kölner Zeitschrift für Soziologie und Sozialpsychologie 1982, 4: 717-731.

Esser, Hartmut, Soziologie. Spezielle Grundlagen. Band 2. Frankfurt/M. 2000.

Esser, Hartmut, Kulturelle Pluralisierung und strukturelle Assimilation: das Problem der ethnischen Schichtung, in: Swiss Political Science Review 2001, 7/2: 97-108.

Frindte, Wolfgang; Boehnke, Klaus; Kreikenborn, Henry und Wagner, Wolfgang, Lebenswelten junger Muslime in Deutschland. Ein sozial- und medienwissenschaftliches System zur Analyse, Bewertung und Prävention islamistischer Radikalisierungsprozesse junger Menschen in Deutschland. Im Auftrag des Bundesministeriums des Inneren, Berlin 2011.

Hans, Silke, Assimilation oder Segregation? Anpassungsprozesse von Einwanderern in Deutschland. Wiesbaden 2010.

Haug, Sonja; Müssig, Stefanie und Stichs, Anja, Muslimisches Leben in Deutschland - im Auftrag der Deutschen Islam Konferenz, in Bundesamt für Migration und Flüchtlinge (BAMF) (Hrsg.), Forschungsbericht 6, Nürnberg 2009.

\footnotetext{
${ }^{24}$ Vgl. dazu auch Martina Sauer und Dirk Halm 2009.

${ }^{25}$ Vgl. Norbert F. Wiley 1967.

${ }^{26}$ Vgl. Georg Elwert 1982.
} 
Portes, Alejandro und Min Zhou, The New Second Generation: Segmented Assimilation and Its Variants. In: Annals of the American Academy of Political and Social Sciences 1993, 530: 74-96.

Putnam, Robert D. (Hrsg.), Gesellschaft und Gemeinsinn. Sozialkapital im internationalen Vergleich. Gütersloh 2001.

Putnam, Robert D., Einleitung. In: Putnam, Robert D. (2001), S. 15-43.

Putnam, Robert D., Bowling Alone. The Collapse and Revival of American Community, New York 2000.

Putnam, Robert D., Turning In, Turning Out: The Strange Disappearance of Social Capital in America. In: Political Science and Politics 1995, 28: 664-683.

Ramadan, Tariq, Muslimsein in Europa. Untersuchung der islamischen Quellen im europäischen Kontext, Marburg 2001.

Roy, Olivier, Der islamische Weg nach dem Westen. Globalisierung, Entwurzelung, Radikalisierung, München 2006.

Sauer, Martina und Dirk Halm, Erfolge und Defizite der Integration türkeistämmiger Einwanderer. Entwicklungen der Lebenssituation 1999 bis 2008, Wiesbaden 2009.

Schiffauer, Werner, Neo-Osmanismus und Hybridität - zwei Varianten der postislamistischen Suche in der Islamischen Gemeinschaft Milli Görüs (IGMG), in: Rauf Ceylan (Hrsg.), Islam und Diaspora. Analysen zum muslimischen Leben in Deutschland aus historischer, rechtlicher sowie migrations- und religionssoziologischer Perspektive, Reihe für Osnabrücker Islamstudien, Band 8, Frankfurt a. M. 2012: 285-296.

Schiffauer, Werner, Nach dem Islamismus. Eine Ethnographie der Islamischen Gemeinschaft Milli Görüs. Berlin 2010.

Wiley, Norbert F., The Ethnic Mobility Trap and Stratification Theory, in: Social Problems 1967, 15/2: 147-159.

Zhou, Min, Segmented Assimilation: Issues, Controversies, and Recent Research on the New Second Generation. In: International Migration Review 1997, 31/4: 975-1008.

\section{Internetquellen}

Lukka, Priya (2003): Energy for Change?: How Faith-based Volunteering is Developing Social Capital and Community Life in the UK, http://66.165.155.20/docs/events/2003_ spring_research_conference/lukka.pdf (Stand: 12.09.2012)

Thießen, Friedrich und Mathias Saggau, Starke Förderung durch islamische Regierungen Islamic Banking ist ein sehr wachstumsstarker Finanzmarkt, in: Betriebswirtschaftliche Blätter 1, 2009, http://www.tu-chemnitz.de/wirtschaft/bwl4/interessantes/IslamicBan king.pdf (Stand 12.09.2012). 
